\title{
miR-152-3p impedes the malignant phenotypes of hepatocellular carcinoma by repressing roundabout guidance receptor 1
}

Tao Yin * and Haonan Zhao

*Correspondence:

tao_y717@163.com

Department of General

Surgery, Affiliated Hospital

of Chifeng University, No. 42

Wangfu Street, Songshan,

Chifeng 024005, China

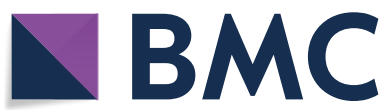

(C) The Author(s) 2022. Open Access This article is licensed under a Creative Commons Attribution 4.0 International License, which permits use, sharing, adaptation, distribution and reproduction in any medium or format, as long as you give appropriate credit to the original author(s) and the source, provide a link to the Creative Commons licence, and indicate if changes were made. The images or other third party material in this article are included in the article's Creative Commons licence, unless indicated otherwise in a credit line to the material. If material is not included in the article's Creative Commons licence and your intended use is not permitted by statutory regulation or exceeds the permitted use, you will need to obtain permission directly from the copyright holder. To view a copy of this licence, visit http:// creativecommons.org/licenses/by/4.0/.
Results: Up-regulation of $R O B O 1$ functioned as an oncogene associated with poor prognosis, immune cell enrichment and cell proliferation in HCC. ROBO1 was significantly positively correlated with the enrichment of multiple immune cells and their biomarkers. Enrichment of type-2 T-helper (Th2) cells is an unfavourable biomarker of HCC prognosis. GSEA revealed that $R O B O 1$ correlated with apoptosis, mitosis and carcinogenic signalling pathways. Suppression of cell proliferation and the enhancement of cell apoptosis by miR-152-3p mimics were counteracted by overexpression of $R O B O 1$ in HCC cells.

Conclusion: $R O B O 1$ expression is positively correlated with multiple immune checkpoint molecules, suggesting that $R O B O 1$ may be a potential drug target to enhance the potency of immunotherapy. The miR-152-3p/ROBO1 signalling axis contributes to malignant progression and provides a prospective immunotherapeutic target for HCC.

Keywords: Roundabout guidance receptor 1, Prognosis, miR-152-3p, Immunotherapy, Hepatic tumorigenesis

\section{Introduction}

The biological functions of miR-152-3p are contradictory to the current understanding of the carcinogenesis of multiple malignant tumours [1-3]. miR-152-3p is recognized as an oncosuppressor in breast cancer [1], prostate cancer [3], colorectal cancer $[4,5]$ and 
glioma [6, 7]. Conversely, up-regulation of miR-152-3p is revealed in plasma from prostate cancer patients compared with healthy control subjects [2]. In our previous study [8], miR-152-3p repressed cyclin-dependent kinase 8 to restrain hepatic carcinogenesis. Herein, we further explored the roles of miR-152-3p and its gene target in the prognosis and immune infiltration of HCC.

$R O B O 1$ is a member of the roundabout transmembrane protein receptor family and contributes to axonal guidance in neurogenesis $[9,10]$. Recently, ROBO proteins have been implicated in tumour angiogenesis, endothelial cell migration and immune cell recruitment, interacting with Slit2 as a corresponding ligand $[11,12]$. The SLIT/ROBO signalling pathway exhibits Janus-faced properties in cancer progression [9]. In several studies, the Slit2/ROBO1 axis restrains the malignant phenotypes, such as migration, invasion and epithelial-mesenchymal transition, of cancer cells [13-15]. However, upregulation of $R O B O 1$ is correlated with poor prognosis and accelerates osteosarcoma cell growth [16]. Moreover, $R O B O 1$ expression is elevated in nasopharyngeal cancer and is associated with worse overall survival [17]. In HCC, ROBO1 is up-regulated in tumour tissues and is one of the poor-prognosis-related and immune-related genes that may contribute to hepatic carcinogenesis $[18,19]$. ROBO1 is also substantiated as a serologic marker for the diagnosis of HCC [19].

In our study, miR-152-3p was up-regulated in nine cancer types and down-regulated in five cancer types in a pan-cancer analysis of the TCGA database. Compared with nontumour tissues, the elevation of $R O B O 1$ and the reduction of miR-152-3p were observed in HCC tissues. Bioinformatics prediction and experimental measurements validated that $R O B O 1$ is a direct gene target of miR-152-3p that can repress the protein expression of $R O B O 1$ in HCC cells. We further investigated the antineoplastic activity, prognosis and immune infiltration of the miR-152-3p/ROBO1 axis in HCC.

\section{Materials and methods}

\section{Prediction of miR-152-3p-related gene targets}

Three miRNA prediction databases, TargetScan, miRDB and RNA22, were used to predict miR-152-3p-related gene targets. A Venn diagram was utilized to visualize the potential gene targets with $\mathrm{R}$ software and the ggplot2 package (version 3.3.3).

\section{TCGA data analysis}

The expression profiles of miR-152-3p and $R O B O 1$ in pan-cancer or HCC were evaluated using the TCGA database with the ggplot2 package (version 3.3.3). The prognostic analysis was carried out using the TCGA database with the survminer package (version 0.4.9) and the survival package (version 3.2-10) as described previously [20]. In addition, the HCCDB database and the HPA database were used to evaluate $R O B O 1$ gene expression and immunostaining in $\mathrm{HCC}$ tissues, respectively.

\section{Prognostic analysis of $\mathrm{ROBO} 1$}

The Kaplan-Meier Plotter database (https://kmplot.com/analysis/index.php?p=backg round) was used to analyse the prognostic significance of $R O B O 1$ in HCC. In addition, to investigate whether $R O B O 1$ is an unfavourable factor for OS by mediating the 
enrichment of immune cells, the Kaplan-Meier Plotter database was used to evaluate OS in the subgroups with enriched and decreased immune cells.

\section{Immune infiltration}

The association between the enrichment of immune cells and $R O B O 1$ expression in HCC was analysed using the GSVA package (version 1.34.0) with the ssGSEA algorithm [21, 22]. In addition, the correlation of $R O B O 1$ with immune checkpoint molecules [cytotoxic T-lymphocyte associated protein $4(C T L A-4)$, programmed cell death 1 (PDCD-1), $C D 274$, T-cell immunoreceptor with Ig and ITIM domains (TIGIT), butyrophilin subfamily 2 member A1 (BTN2A1) and BTN2A2] was assessed in the TCGA database using the ggplot2 package (version 3.3.3).

\section{GSEA, GO and KEGG pathway enrichment}

An analysis of single-gene differences of $R O B O 1$ in the TCGA database was prepared for GSEA using the DESeq2 package (version 1.26.0) as described previously [23]. In addition, GO and KEGG pathway enrichment analyses were predicted by the DAVID online database (https://david.ncifcrf.gov/).

\section{Cell experiments}

Cell experiments, including cell culture, cell transfection, luciferase reporter assays (Promega, USA), western blotting (anti-ROBO1: ab7279; dilution: 1:500; Abcam) and CCK8 assays (Beyotime), were carried out as described previously [8]. miR-Com-, miR152-3p-, sh-Con-, sh-ROBO1- and ROBO1-overexpressing plasmids were obtained from GenePharma (Shanghai, China). A TUNEL kit (Roche) was utilized to analyse cell apoptosis.

\section{Statistical analysis}

The data were analysed using Mann-Whitney $U$ test, Wilcoxon signed rank test, oneway analysis of variance log-rank test, univariate Cox regression analysis and Spearman's correlation analysis.

\section{Results}

\section{Prediction of gene targets of miR-152-3p}

Based on three miRNA prediction databases, TargetScan, miRDB and RNA22, a total of 101 gene targets of miR-152-3p were collectively identified in those three databases (Fig. 1A). The heatmap shown in Fig. 1B presents the differential expression profiles of the 101 gene targets in the TCGA database, which contained 160 non-tumour tissues and $371 \mathrm{HCC}$ specimens. According to $\log _{2}$ (fold change) $>2$ and $p<0.05, R O B O 1$ $\left[\log _{2}(\right.$ fold change $\left.)=2.21 ; p<0.001\right]$ and COL4A1 $\left[\log _{2}(\right.$ fold change $\left.)=2.07 ; p<0.001\right]$ expression levels were elevated in HCC tissues and filtered out for further investigations (Fig. 1C). In 50 paired tissues, both $R O B O 1$ and $C O L 4 A 1$ were expressed at significantly higher levels in cancerous tissues than in paracarcinoma tissues (Fig. 1D). 


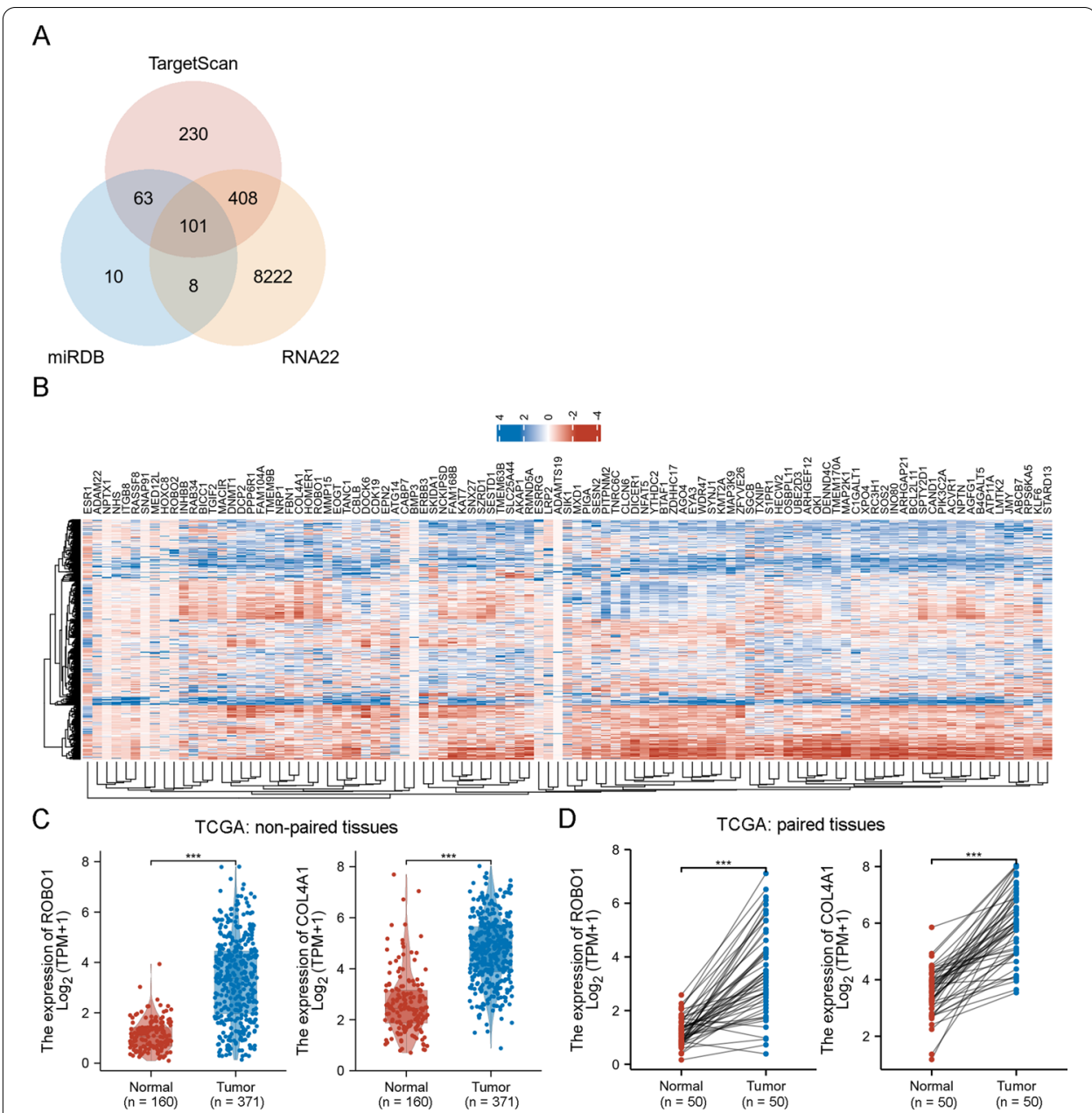

Fig. 1 Prediction of gene targets of miR-152-3p. TargetScan, miRDB and RNA22 were implemented to predict gene targets of miR-152-3p (A). Heatmap represents the differential expression of 101 gene targets (B). $R O B O 1$ and $C O L 4 A 1$ expression in non-paired HCC tissues and non-tumour tissues (C). ROBO1 and COL4A1 expression in paired HCC tissues and adjacent tissues (D). ${ }^{* * *} p<0.001$

\section{Prognostic significance of $R O B O 1$ and COL4A1 in HCC}

The Kaplan-Meier Plotter database was used to evaluate the association of $R O B O 1$ and COL4A1 with prognosis in HCC. Worse OS, PFS and DSS were observed in patients with high $R O B O 1$ expression than in those with low ROBO1 expression. However, COL4A1 expression had no significant correlation with OS, RFS, PFS or DSS in HCC patients (Fig. 2B).

\section{Further validation of $R O B O 1$ expression in $\mathrm{HCC}$}

The HCCDB database was utilized to analyse $R O B O 1$ gene expression in 12 datasets. In 11 of the 12 datasets, up-regulation of $R O B O 1$ gene expression in $\mathrm{HCC}$ tissues was validated by the HCCDB database (Fig. 3A). Intriguingly, the HPA database revealed prominent positive staining of $R O B O 1$ expression in HCC tissues (Fig. 3B). As shown in Fig. $3 \mathrm{C}$ and Table 1, ROBO1 expression was not significantly different in the T, N, M and 

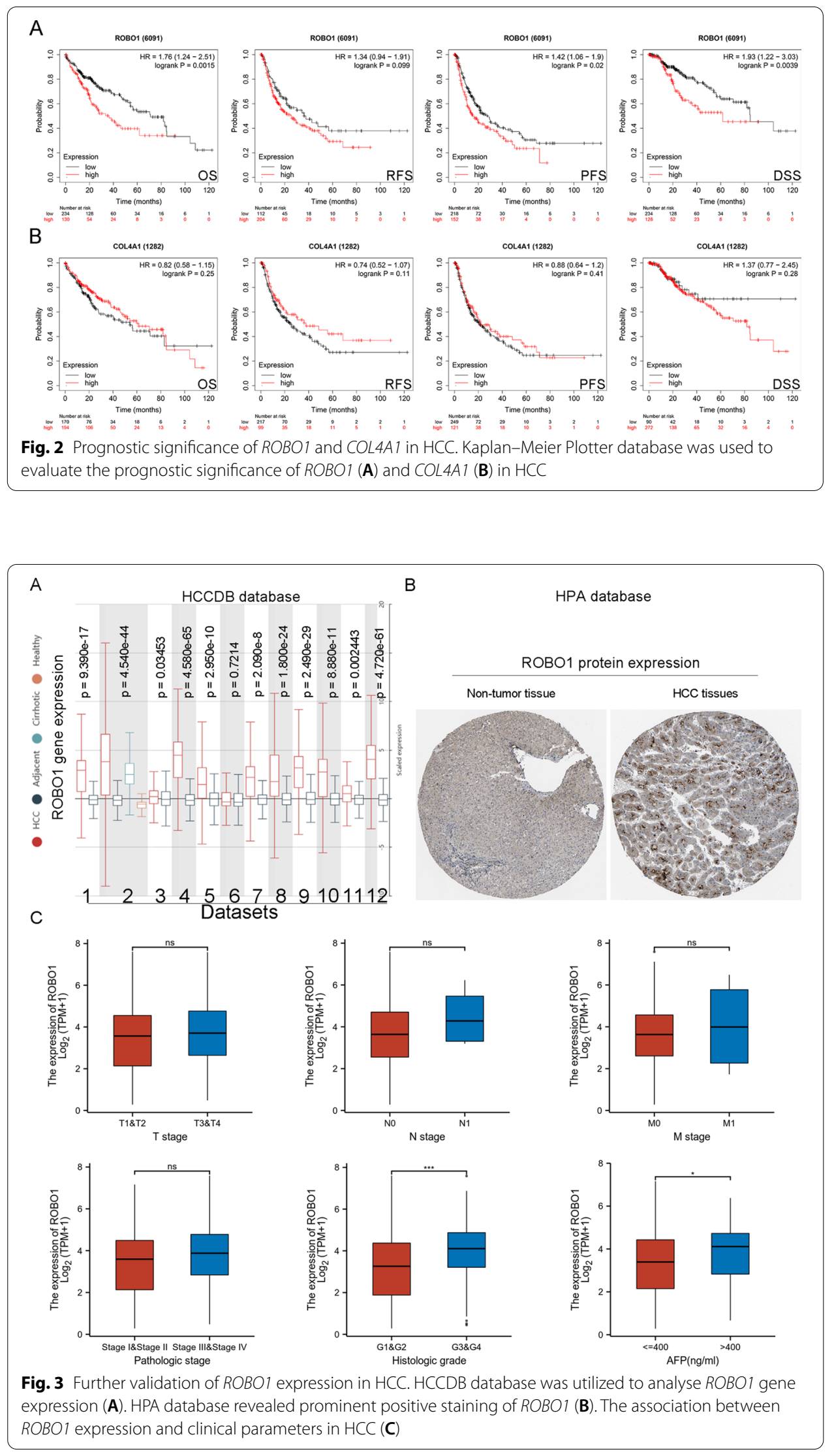
Table 1 The association of ROBO1 expression with clinical parameters in HCC patients

\begin{tabular}{|c|c|c|c|}
\hline Characteristic & Low expression of ROBO1 & $\begin{array}{l}\text { High expression of } \\
\text { ROBO1 }\end{array}$ & $p$ \\
\hline$n$ & 187 & 187 & \\
\hline Age, $n(\%)$ & & & 0.797 \\
\hline$\leq 60$ & $87(23.3 \%)$ & $90(24.1 \%)$ & \\
\hline$>60$ & $100(26.8 \%)$ & $96(25.7 \%)$ & \\
\hline Gender, $n(\%)$ & & & 0.507 \\
\hline Female & $64(17.1 \%)$ & $57(15.2 \%)$ & \\
\hline Male & $123(32.9 \%)$ & $130(34.8 \%)$ & \\
\hline Race, $n(\%)$ & & & 0.617 \\
\hline Asian & $76(21 \%)$ & $84(23.2 \%)$ & \\
\hline Black or African American & $10(2.8 \%)$ & $7(1.9 \%)$ & \\
\hline White & $94(26 \%)$ & $91(25.1 \%)$ & \\
\hline Pathologic stage, $n(\%)$ & & & 0.384 \\
\hline Stage I & 91 (26\%) & $82(23.4 \%)$ & \\
\hline Stage II & $40(11.4 \%)$ & 47 (13.4\%) & \\
\hline Stage III & $36(10.3 \%)$ & 49 (14\%) & \\
\hline Stage IV & $3(0.9 \%)$ & $2(0.6 \%)$ & \\
\hline Tstage, $n(\%)$ & & & 0.563 \\
\hline $\mathrm{T} 1$ & $97(26.1 \%)$ & $86(23.2 \%)$ & \\
\hline $\mathrm{T} 2$ & $44(11.9 \%)$ & $51(13.7 \%)$ & \\
\hline T3 & $38(10.2 \%)$ & $42(11.3 \%)$ & \\
\hline $\mathrm{T} 4$ & $5(1.3 \%)$ & $8(2.2 \%)$ & \\
\hline N stage, $n(\%)$ & & & 1.000 \\
\hline No & $124(48.1 \%)$ & $130(50.4 \%)$ & \\
\hline N1 & $2(0.8 \%)$ & $2(0.8 \%)$ & \\
\hline M stage, $n(\%)$ & & & 1.000 \\
\hline Mo & $132(48.5 \%)$ & $136(50 \%)$ & \\
\hline M1 & $2(0.7 \%)$ & $2(0.7 \%)$ & \\
\hline Histologic grade, $n$ (\%) & & & $<0.001$ \\
\hline G1 & 39 (10.6\%) & $16(4.3 \%)$ & \\
\hline G2 & 95 (25.7\%) & $83(22.5 \%)$ & \\
\hline G3 & 45 (12.2\%) & $79(21.4 \%)$ & \\
\hline G4 & $4(1.1 \%)$ & $8(2.2 \%)$ & \\
\hline AFP (ng/ml), n (\%) & & & 0.012 \\
\hline$\leq 400$ & $123(43.9 \%)$ & $92(32.9 \%)$ & \\
\hline$>400$ & 25 (8.9\%) & $40(14.3 \%)$ & \\
\hline Age, median (IQR) & $61(52,69)$ & $61(51.25,68)$ & 0.486 \\
\hline
\end{tabular}

pathologic stage subgroups. However, up-regulation of $R O B O 1$ showed significant correlations with high histologic grade and AFP levels in HCC patients.

\section{Relationship of $R O B O 1$ expression with immune infiltration and immune checkpoint molecules}

To evaluate whether $R O B O 1$ expression was associated with tumour immunity, the ssGSEA algorithm was implemented to investigate whether $R O B O 1$ expression mediates immune cell enrichment in HCC tissues. Spearman correlation analysis revealed that $R O B O 1$ was positively correlated with five immune cell enrichments and negatively 
correlated with six immune cell enrichments in HCC tissues (Fig. 4A). The top three correlated immune cells, including T-helper cells $(r=0.329 ; p<0.001)$, DCs $(r=-0.203$; $p<0.001)$ and cytotoxic cells $(r=-0.190 ; p<0.001)$, are listed in Fig. 4B and C. In addition, $R O B O 1$ was positively correlated with five immune checkpoint molecules (Fig. 4D), including PDCD1 ( $r=0.170 ; p=0.001)$, CTLA4 $(r=0.220 ; p<0.001)$, TIGIT $(r=0.190$; $p<0.001), B T N 2 A 1(r=0.320 ; p<0.001)$ and BTN2A2 $(r=0.260 ; p<0.001)$.

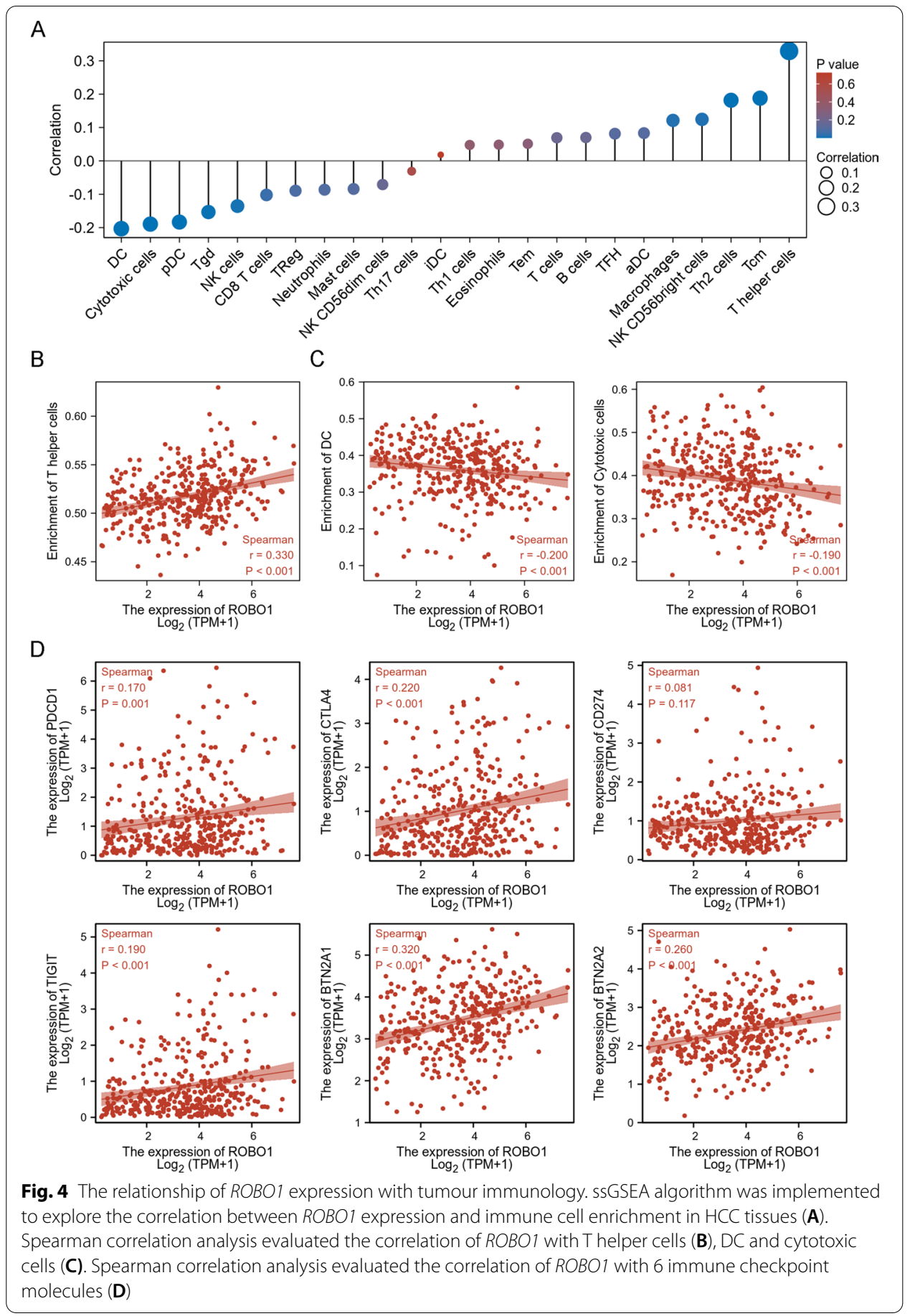




\section{Prognostic significance of $R O B O 1$ expression based on immune cell enrichment in HCC patients}

The TIMER database was also used to validate the association between $R O B O 1$ expression and immune cell enrichment in HCC. As shown in Fig. 5A, ROBO1 expression was positively correlated with B cells $\left(r=0.154 ; p=4.31 \times 10^{-3}\right)$, CD4 ${ }^{+} \mathrm{T}$ cells $(r=0.244$; $\left.p=4.55 \times 10^{-6}\right)$, macrophages $\left(r=0.176 ; p=1.11 \times 10^{-3}\right)$, neutrophils $(r=0.202$; $\left.p=1.56 \times 10^{-4}\right)$ and dendritic cells $\left(r=0.116 ; p=3.25 \times 10^{-2}\right)$. Based on ROBO1, in the above-mentioned results, high $R O B O 1$ expression was correlated with poor prognosis and immune cell enrichment. Therefore, we hypothesized that the ROBO1-regulated

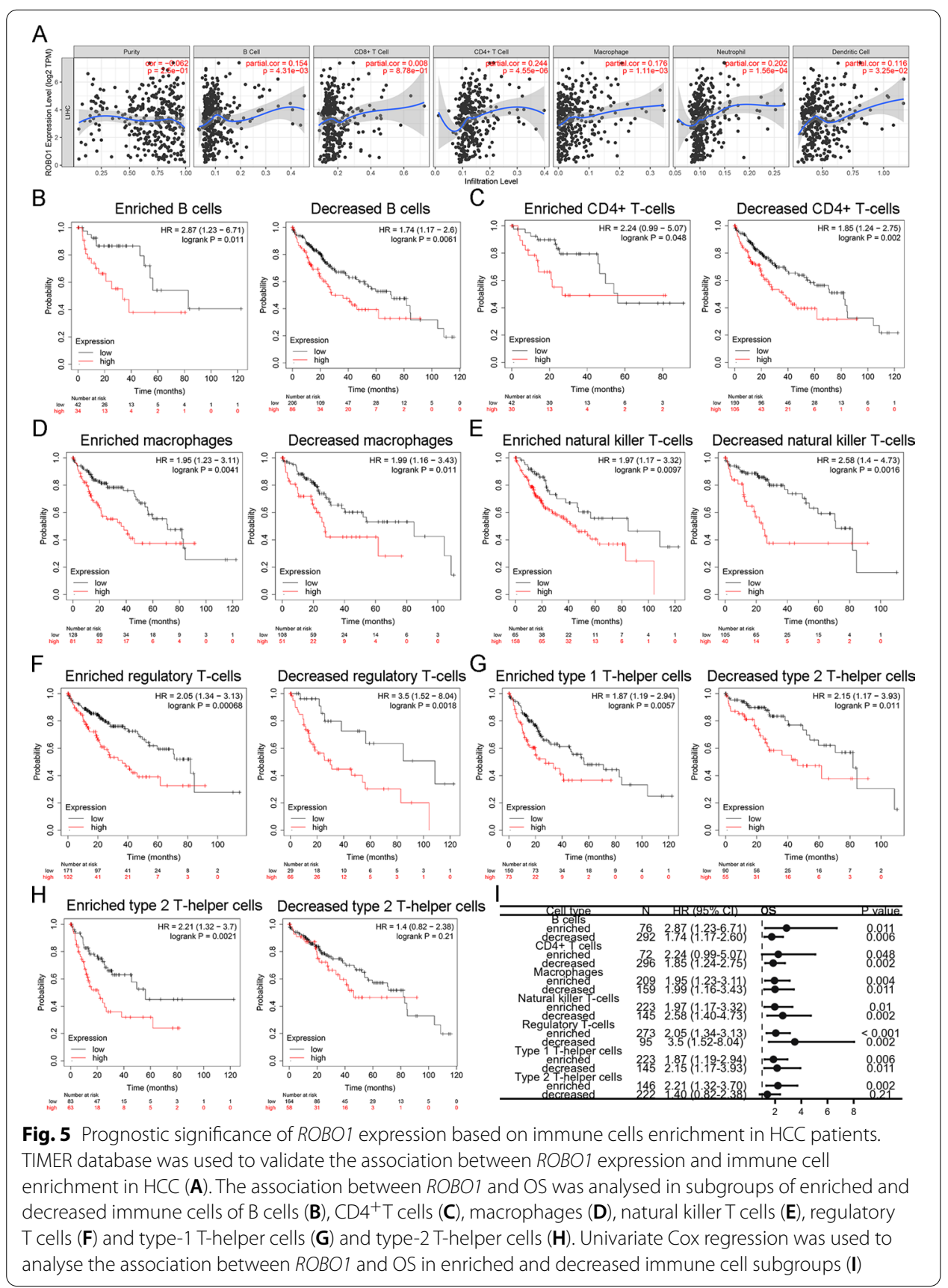


poor prognosis was associated with immune cell enrichment. The association between $R O B O 1$ and $O S$ was analysed in the subgroups with enriched and decreased immune cells. In both the enriched and decreased subgroups of B cells (Fig. 5B, I), CD4 ${ }^{+} \mathrm{T}$ cells (Fig. 5C, I) macrophages (Fig. 5D, I), natural killer T cells (Fig. 5E, I), regulatory T cells (Fig. 5F, I) and type-1 T-helper cells (Fig. 5G, I), high ROBO1 expression was significantly correlated with poor OS in HCC patients. As shown in Fig. $5 \mathrm{H}$ and I, high $R O B O 1$ expression correlated with poor OS in HCC patients with enriched type-2 T-helper cells, suggesting that poor ROBO1-related OS may be partially mediated by the enrichment of type-2 T-helper cells. To further validate the association of $R O B O 1$ with immune cell enrichment in $\mathrm{HCC}$, the correlation between $\mathrm{ROBO1}$ and multiple biomarkers of immune cells was evaluated using TCGA database. As shown in Table 2, ROBO1 was significantly positively correlated with T-helper cell biomarkers (CXCR3, CCR4, CCR6 and CCR10), Tcm biomarkers (CD62L, CCR5, CD58 and TCF7), Th2 cell biomarkers (PTGDR2, IL5 and IL10) and macrophage biomarkers (PPARG, IRF5 and CD68) in HCC.

\section{Differentially expressed genes in $\mathrm{HCC}$ based on $\mathrm{ROBO} 1$ expression}

According to $\mid \log _{2}$ (fold change) $\mid>2$ and adjusted $p<0.05,132$ up-regulated and 68 downregulated genes were filtered based on the $R O B O 1$ high- and low-expression subgroups (Fig. 6A). A heatmap was constructed to visualize the expression profiles of the top five up-regulated and down-regulated genes in HCC tissues (Fig. 6B). GSEA suggested that ROBO1-related genes were enriched in the Biocarta intrinsic pathway, Reactome CD22 mediator BCR regulation, the PID/PLK1 pathway, Reactome mitotic prometaphase, the PID/MYC activity pathway and the PID/beta-catenin Nuc pathway (Fig. 6C).

\section{GO and KEGG analyses}

The $R O B O 1$-related top 100 up-regulated and down-regulated genes were used to perform GO and KEGG analyses. Seven BP terms (Fig. 7A), 15 MF terms (Fig. 7B) and 7 KEGG pathways (Fig. 7C) were enriched in HCC.

Table 2 The association of ROBO1 expression with the biomarkers of immune cells

\begin{tabular}{lllr}
\hline Immune cell & Biomarker & $\boldsymbol{r}$ value & $\boldsymbol{p}$ value \\
\hline T-helper cell & CXCR3 & 0.167 & 0.001 \\
& CCR4 & 0.222 & $<0.001$ \\
& CCR6 & 0.327 & $<0.001$ \\
CCR10 & 0.148 & 0.004 \\
Tcm & 0.183 & $<0.001$ \\
& CD62L & 0.201 & $<0.001$ \\
CCR5 & 0.354 & $<0.001$ \\
CD58 & 0.305 & $<0.001$ \\
Th2 cells & TCF7 & 0.228 & $<0.001$ \\
& PTGDR2 & 0.162 & 0.002 \\
IL5 & 0.181 & $<0.001$ \\
Macrophage & IL10 & 0.379 & $<0.001$ \\
& PPARG & 0.224 & $<0.001$ \\
& IRF5 & 0.179 & $<0.001$ \\
\hline
\end{tabular}




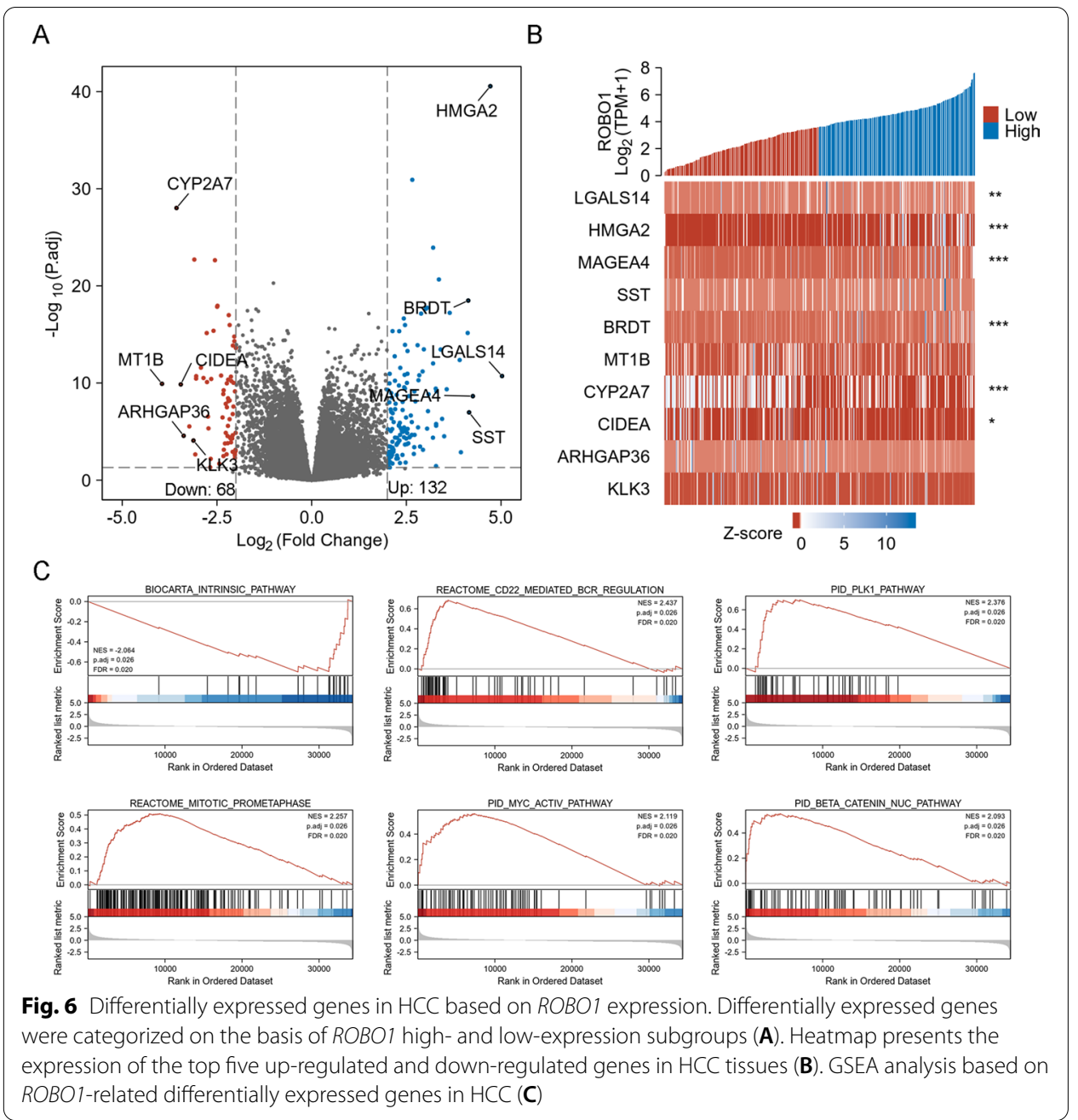

\section{Validation of $R O B O 1$ as a gene target of miR-152-3p}

The analyses performed in the online databases revealed that $R O B O 1$ might be a potential gene target of miR-152-3p, which was predicted to bind with the $3^{\prime}$-UTR of ROBO1 (Fig. 8A). In vitro luciferase activity was significantly diminished in HepG2 and Huh7 cells after transfection with miR-152-3p mimics compared with the control group (Fig. 8B), indicating that miR-152-3p can directly bind with ROBO1. Western blot analysis revealed significant decreases in $R O B O 1$ protein expression in HepG2 and Huh7 cells transfected with miR-152-3p mimics (Fig. 8C). To investigate the roles of miR-152-3p and $R O B O 1$ in the progression of HCC, miR-152-3p mimics, sh- $R O B O 1$ or $R O B O 1$ overexpression plasmids were delivered into HepG2 and Huh7 cells. As shown in Fig. 8D and E, transfection of miR-152-3p mimics or sh-ROBO1 inhibited cell proliferation and induced cell apoptosis of HepG2 and Huh7 cells. However, the inhibition of cell proliferation and the enhancement of cell apoptosis by miR-152-3p mimics were counteracted by overexpression of ROBO1 in HepG2 and Huh7 cells (Fig. 8D, E). 


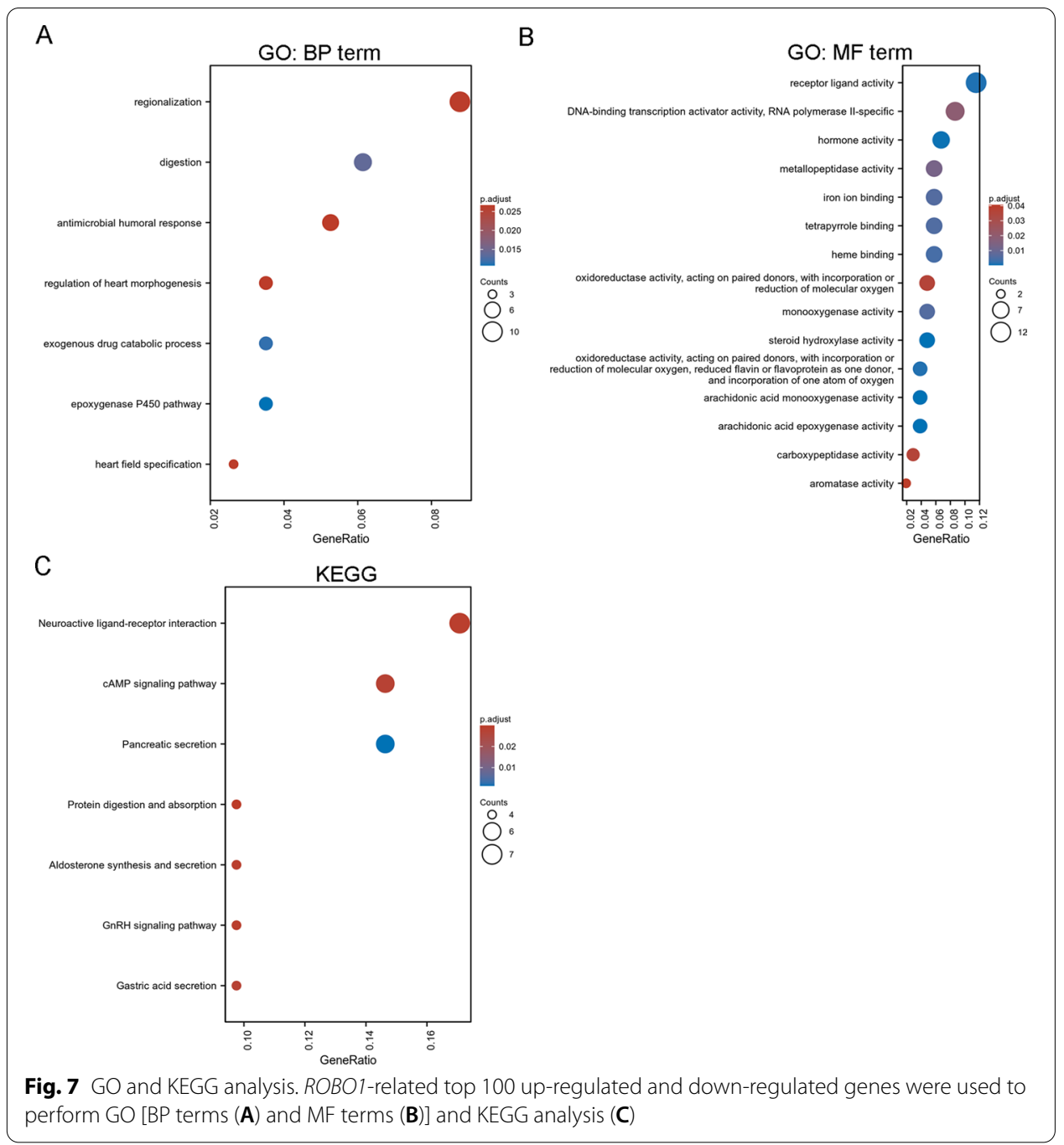

The association between miR-152-3p and HCC

As shown in Fig. 9A, miR-152-3p was up-regulated in nine cancer types and downregulated in five cancer types in pan-cancer analysis of the TCGA database. Compared with non-tumour tissues, miR-152-3p was significantly down-regulated in HCC tissues (Fig. 9B), while miR-152-3p expression had no significant correlation with OS (Fig. 9C), T stage (Fig. 9D), N stage (Fig. 9E), M stage (Fig. 9F), pathologic stage (Fig. 9G) or histologic grade (Fig. 9H). As shown in Fig. 9I, low miR-152-3p expression was significantly correlated with high AFP levels in HCC patients.

\section{The association between miR-152-3p and immune infiltration}

To determine whether miR-152-3p expression was associated with tumour immunity, the ssGSEA algorithm was implemented to explore the correlation between miR-152-3p expression and immune cell enrichment in HCC tissues. Spearman correlation analysis revealed that $R O B O 1$ was positively correlated with six immune cell enrichments and negatively correlated with two immune cell enrichments in HCC tissues (Fig. 10A). The top 


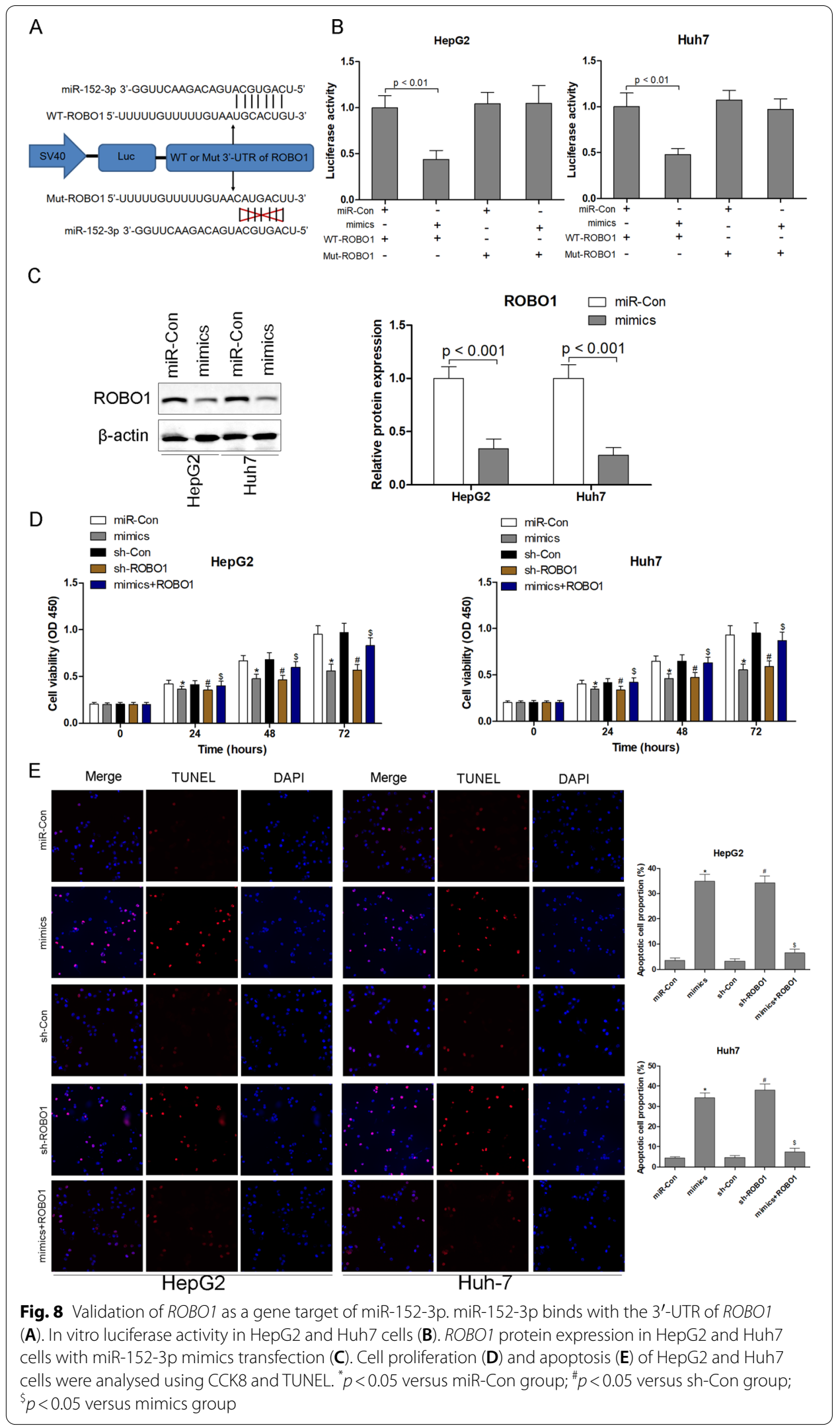




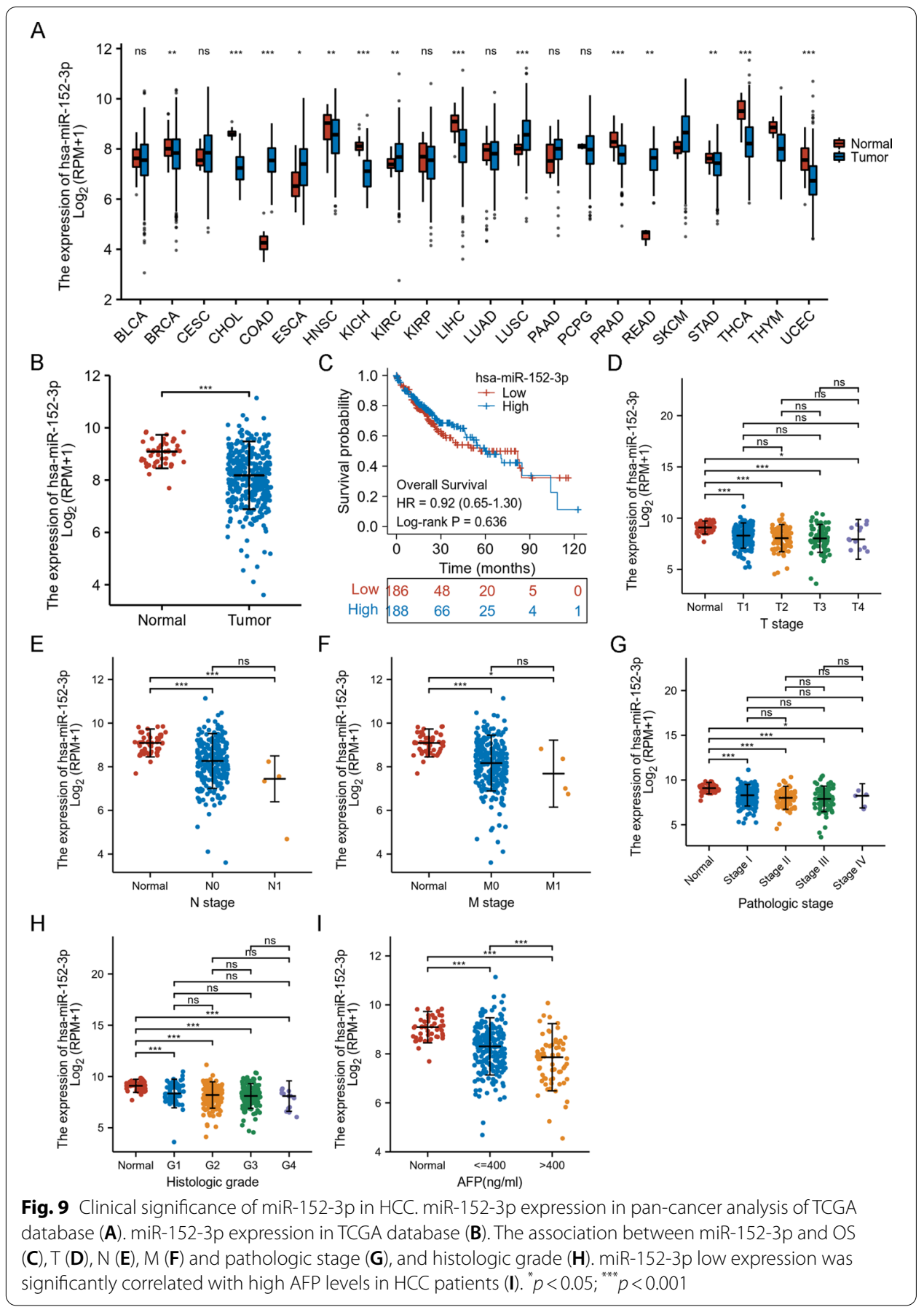

six correlated immune cells, including Th17 cells $(r=0.180 ; p<0.001)$, NK cells $(r=0.180$; $p=0.001)$, DCs $(r=0.170 ; p=0.001)$, mast cells $(r=0.160 ; p=0.002)$, neutrophils $(r=0.140$; $p=0.007)$ and $\operatorname{Tgd}(r=0.130 ; p=0.012)$, are shown in Fig. 10B. 


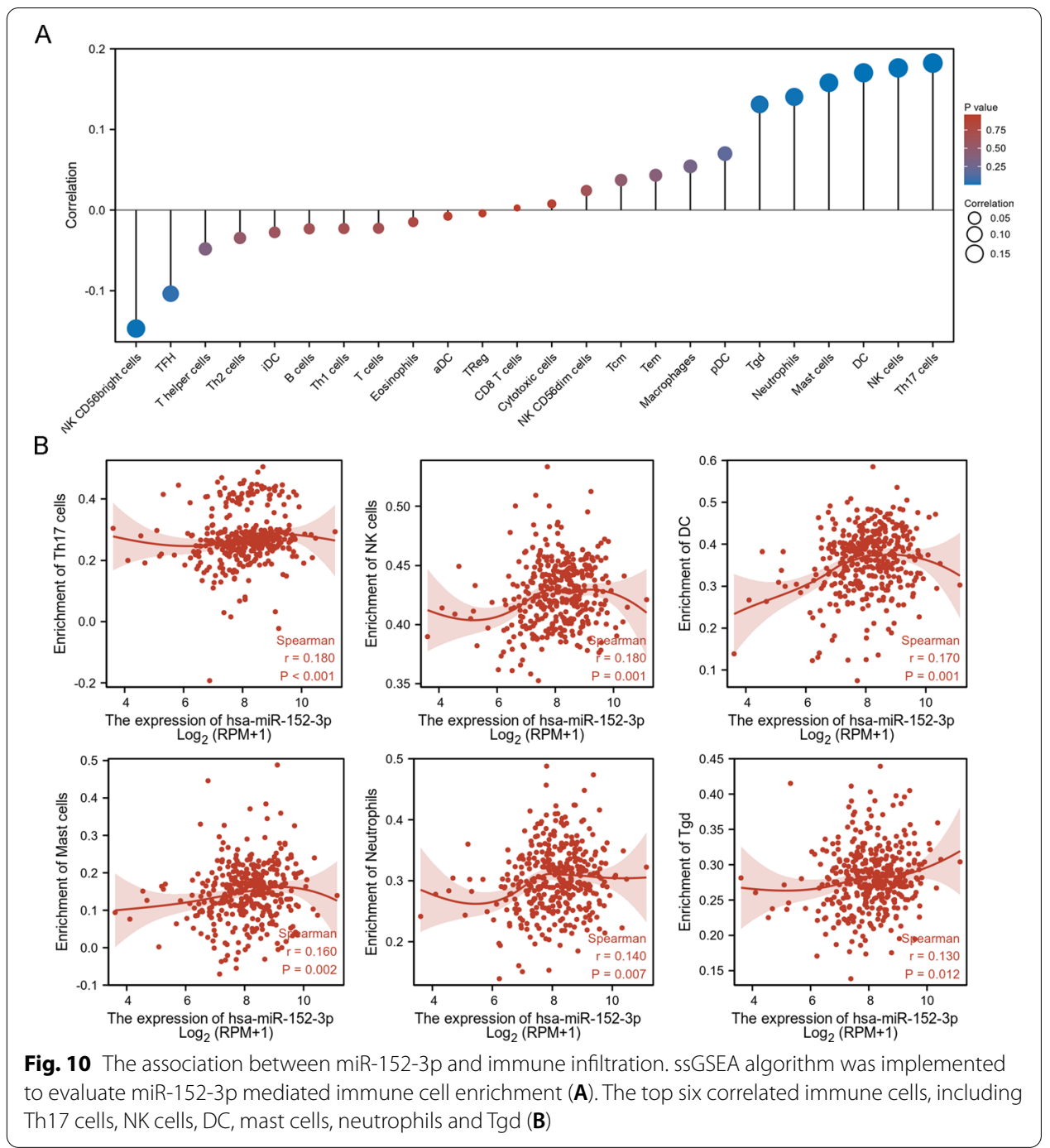

\section{Discussion}

Our findings revealed a novel signalling axis, miR-152-3p/ROBO1, that contributes to hepatic tumorigenesis by regulating cell proliferation and apoptosis in vitro and mediates immune cell enrichment in HCC. ROBO1 was identified as an oncogene to accelerate the enrichment of type-2 T-helper cells that may be correlated with poor prognosis in patients with HCC.

The convertible role of $R O B O 1$ as a tumour suppressor or an oncogene has been observed in different cancer types [15, 24-26]. At present, the transcription level and functions of $R O B O 1$ are consistent in the progression of $\mathrm{HCC}[19,27,28]$. For example, the enhancement of $R O B O 1$ expression triggers tumour growth, invasion and metastasis in HCC Sk-hep-1 cells [27]. Down-regulation of ROBO1 by miR-490-5p contributes to the induction of apoptosis and inhibits malignant phenotypes in HCC Hep3B cells [28]. Hirotaka et al. substantiated that $\mathrm{ROBO1}$ is overexpressed in the serum of HCC patients, HCC tissues and cell lines and may be a serologic marker for the diagnosis of HCC [19]. In our study, marked elevations in $R O B O 1$ gene and protein expression 
were corroborated by three databases, TCGA, HCCDB and HPA, in HCC tissues, and $R O B O 1$, as an unfavourable prognostic marker, was correlated with worse OS, PFS and DSS. We also found that $R O B O 1$ loss of function augmented the proportion of apoptotic cells and restrained the proliferation of HCC cell lines.

Diversiform immune cells are an important component of the tumour microenvironment and correlate with prognosis, metastasis and immunotherapy in cancer [29, 30]. $\mathrm{HCC}$ is associated with inflammatory processes, such as viral infection and cirrhosis, which drive the enrichment of immune cells, especially lymphocytes, contributing to poor prognosis [30, 31]. In our study, both the TCGA and TIMER databases indicated that $R O B O 1$ was significantly and positively correlated with macrophages in HCC tissues. Tumour-associated macrophages (TAMs) contribute to the initiation and progression of HCC by secreting pro-inflammatory cytokines and triggering the expansion of cancer stem cells [32]. In addition, TAMs facilitate metastasis and predict poor prognosis in patients with HCC [32, 33].

Our findings also suggested that poor OS in $R O B O 1 \mathrm{HCC}$ patients with high $R O B O 1$ expression was associated with the enrichment of Th2 cells. The TCGA database also validated a significant positive correlation $(r=0.182 ; p<0.001)$ between $R O B O 1$ and Th2 cells in HCC tissues. Enrichment of Th2 cells has frequently been reported in HCC patients and is implicated in tumour invasion and metastasis $[34,35]$. Duan et al. indicated that inhibition of Th2 cell activity by immune checkpoint blockades (ICBs) may be associated with increased survival time and decreased tumour recurrence in a mouse model of HCC [36]. These findings suggested that enrichment of Th2 cells represented an unfavourable biomarker of HCC prognosis.

ICBs are a class of antitumour immunotherapeutic drugs that suppress immune checkpoint molecules, such as PD-1, PD-L1, CTLA-4 and TIGIT, to restore immune recognition and immunogenicity in HCC [37-39]. Our findings suggested that ROBO1 was positively correlated with five immune checkpoint molecules, PDCD1, CTLA4, TIGIT, $B T N 2 A 1$ and $B T N 2 A$, in $\mathrm{HCC}$, reflecting that $R O B O 1$ inhibitors may have a synergistic effect and enhance the potency of ICBs to improve therapeutic efficiency in HCC patients.

In this study, both predictions and experiments corroborated that $R O B O 1$ is a direct gene target of miR-152-3p. In our previous study [8], miR-152-3p expression was decreased in HCC tissues, and overexpression of miR-152-3p targeted cyclin-dependent kinase 8 to mediate antineoplastic activity in HCC. Other studies have also shown that miR-152-3p possesses outstanding anticancer properties in colorectal cancer, prostate cancer and lymphoma [5, 40,41]. Based on our previous findings [8] and the present results, miR-152-3p may function as a tumour suppressor by mediating multiple gene targets to prevent hepatic tumorigenesis.

In conclusion, $R O B O 1$ was identified as an unfavourable prognostic marker and was correlated with the enrichment of Th2 cells in HCC. ROBO1 expression was also positively correlated with multiple immune checkpoint molecules, suggesting that $R O B O 1$ may be a potential drug target to enhance the potency of immunotherapy. Further, $R O B O 1$ was identified as a direct target of miR-152-3p, indicating that the miR-152$3 \mathrm{p} / \mathrm{ROBO1}$ signalling axis may be involved in the pathogenesis of hepatic tumorigenesis. 


\section{Abbreviations}

HCC: Hepatocellular carcinoma; TCGA: The Cancer Genome Atlas; HCCDB: Integrative molecular database of hepatocellular carcinoma; HPA: Human Protein Atlas; ROBO1: Roundabout guidance receptor 1; CCK-8: Cell Counting Kit 8; TUNEL: Terminal-deoxynucleotidyl transferase-mediated nick end labelling; Th2: Type 2 T-helper.

\section{Acknowledgements}

Not applicable.

\section{Authors' contributions}

$\mathrm{HZ}$ and TY were responsible for data acquisition and analysis, methodology and manuscript writing. Manuscript was checked by $\mathrm{HZ}$ and TY. Funding was obtained by TY. The manuscript was approved by $\mathrm{HZ}$ and TY to be published. Both the authors read and approved the final manuscript.

\section{Funding}

Our research was supported by the Natural Science Foundation of Inner Mongolia Autonomous Region of China (grant. no: 2020MS08017)

\section{Availability of data and materials}

The data that support the findings of this study are available from the corresponding author upon reasonable request.

\section{Declarations}

Ethics approval and consent to participate

Not applicable.

\section{Consent for publication}

Not applicable.

\section{Competing interests}

The authors declare they have no competing interests.

Received: 23 December 2021 Accepted: 9 February 2022

Published online: 02 March 2022

\section{References}

1. Zeng K, He B, Yang BB, Xu T, Chen X, Xu M, et al. The pro-metastasis effect of circANKS1B in breast cancer. Mol Cancer. 2018;17:160

2. Moya L, Meijer J, Schubert S, Matin F, Batra J. Assessment of miR-98-5p, miR-152-3p, miR-326 and miR-4289 expression as biomarker for prostate cancer diagnosis. Int J Mol Sci. 2019;20:1154.

3. Ramalho-Carvalho J, Gonçalves CS, Graça I, Bidarra D, Pereira-Silva E, Salta S, et al. A multiplatform approach identifies miR-152-3p as a common epigenetically regulated onco-suppressor in prostate cancer targeting TMEM97. Clin Epigenet. 2018;10:40.

4. Wang C, Ma X, Zhang J, Jia X, Huang M. DNMT1 maintains the methylation of miR-152-3p to regulate TMSB10 expression, thereby affecting the biological characteristics of colorectal cancer cells. IUBMB Life. 2020;72:2432-43.

5. Liu X, Li L, Bai J, Li L, Fan J, Fu Z, et al. IncRNA PVT1 promotes progression of colorectal cancer by sponging miR152-3p and regulating E2F3/MAPK8 signaling. Cancer Sci. 2021;113:109-19. https://doi.org/10.1111/cas.15113.

6. Shi J, Zhang Y, Qin B, Wang Y, Zhu X. Long non-coding RNA LINC00174 promotes glycolysis and tumor progression by regulating miR-152-3p/SLC2A1 axis in glioma. J Exp Clin Cancer Res. 2019;38:395.

7. Sun J, Tian $X$, Zhang J, Huang $Y$, Lin $X$, Chen $L$, et al. Regulation of human glioma cell apoptosis and invasion by miR-152-3p through targeting DNMT1 and regulating NF2: MiR-152-3p regulate glioma cell apoptosis and invasion. J Exp Clin Cancer Res. 2017;36:100.

8. Yin T, Liu MM, Jin RT, Kong J, Wang SH, Sun WB. miR-152-3p modulates hepatic carcinogenesis by targeting cyclindependent kinase 8. Pathol Res Pract. 2019;215: 152406.

9. Jiang Z, Liang G, Xiao Y, Qin T, Chen X, Wu E, et al. Targeting the SLIT/ROBO pathway in tumor progression: molecular mechanisms and therapeutic perspectives. Ther Adv Med Oncol. 2019;1 1:1758835919855238.

10. Rama N, Dubrac A, Mathivet T, Chárthaigh RAN, Genet G, Cristofaro B, et al. Slit2 signaling through ROBO1 and Robo2 is required for retinal neovascularization. Nat Med. 2015;21:483-91.

11. Legg JA, Herbert JM, Clissold P, Bicknell R. Slits and roundabouts in cancer, tumour angiogenesis and endothelial cell migration. Angiogenesis. 2008;11:13-21.

12. Koohini Z, Koohini Z, Teimourian S. Slit/Robo signaling pathway in cancer; a new stand point for cancer treatment. Pathol Oncol Res. 2019;25:1285-93.

13. Xia Y, Wang $L, X u Z$, Kong R, Wang F, Yin $K$, et al. Reduced USP33 expression in gastric cancer decreases inhibitory effects of Slit2-ROBO1 signalling on cell migration and EMT. Cell Prolif. 2019;52: e12606.

14. Xu Y, Li WL, Fu L, Gu F, Ma YJ. Slit2/ROBO1 signaling in glioma migration and invasion. Neurosci Bull. 2010;26:474-8

15. Feng Y, Feng L, Yu D, Zou J, Huang Z. srGAP1 mediates the migration inhibition effect of Slit2-ROBO1 in colorectal cancer. J Exp Clin Cancer Res. 2016;35:191.

16. Zhao SJ, Shen YF, Li Q, He YJ, Zhang YK, Hu LP, et al. SLIT2/ROBO1 axis contributes to the Warburg effect in osteosarcoma through activation of SRC/ERK/C-MYC/PFKFB2 pathway. Cell Death Dis. 2018;9:390. 
17. Alajez NM, Lenarduzzi M, Ito E, Hui AB, Shi W, Bruce J, et al. MiR-218 suppresses nasopharyngeal cancer progression through downregulation of survivin and the SLIT2-ROBO1 pathway. Cancer Res. 2011;71:2381-91.

18. Wu L, Quan W, Luo Q, Pan Y, Peng D, Zhang G. Identification of an immune-related prognostic predictor in hepatocellular carcinoma. Front Mol Biosci. 2020;7: 567950.

19. Ito H, Funahashi S, Yamauchi N, Shibahara J, Midorikawa Y, Kawai S, et al. Identification of ROBO1 as a novel hepatocellular carcinoma antigen and a potential therapeutic and diagnostic target. Clin Cancer Res. 2006;12:3257-64.

20. Liu J, Lichtenberg T, Hoadley KA, Poisson LM, Lazar AJ, Cherniack AD, et al. An integrated TCGA pan-cancer clinical data resource to drive high-quality survival outcome analytics. Cell. 2018;173:400-16.e11.

21. Hänzelmann S, Castelo R, Guinney J. GSVA: gene set variation analysis for microarray and RNA-seq data. BMC Bioinform. 2013;14:7.

22. Bindea G, Mlecnik B, Tosolini M, Kirilovsky A, Waldner M, Obenauf AC, et al. Spatiotemporal dynamics of intratumoral immune cells reveal the immune landscape in human cancer. Immunity. 2013;39:782-95.

23. Love MI, Huber W, Anders S. Moderated estimation of fold change and dispersion for RNA-seq data with DESeq2. Genome Biol. 2014;15:550.

24. Parray A, Siddique HR, Kuriger JK, Mishra SK, Rhim JS, Nelson HH, et al. ROBO1, a tumor suppressor and critical molecular barrier for localized tumor cells to acquire invasive phenotype: study in African-American and Caucasian prostate cancer models. Int J Cancer. 2014;135:2493-506.

25. Le LT, Cazares O, Mouw JK, Chatterjee S, Macias H, Moran A, et al. Loss of miR-203 regulates cell shape and matrix adhesion through $R O B O 1 /$ Rac/FAK in response to stiffness. J Cell Biol. 2016;212:707-19.

26. Tie J, Pan Y, Zhao L, Wu K, Liu J, Sun S, et al. MiR-218 inhibits invasion and metastasis of gastric cancer by targeting the $R O B O 1$ receptor. PLoS Genet. 2010;6: e1000879.

27. Yuan M, Guo H, Li J, Sui C, Qin Y, Wang J, et al. Slit2 and ROBO1 induce opposing effects on metastasis of hepatocellular carcinoma Sk-hep-1 cells. Int J Oncol. 2016;49:305-15.

28. Chen W, Ye L, Wen D, Chen F. MiR-490-5p inhibits hepatocellular carcinoma cell proliferation, migration and invasion by directly regulating $R O B O 1$. Pathol Oncol Res. 2019;25:1-9.

29. Ringelhan M, Pfister D, O'Connor T, Pikarsky E, Heikenwalder M. The immunology of hepatocellular carcinoma. Nat Immunol. 2018;19:222-32.

30. Lu C, Rong D, Zhang B, Zheng W, Wang X, Chen Z, et al. Current perspectives on the immunosuppressive tumor microenvironment in hepatocellular carcinoma: challenges and opportunities. Mol Cancer. 2019;18:130.

31. Ally A, Balasundaram M, Carlsen R, Chuah E, Clarke A, Dhalla N, et al. Comprehensive and integrative genomic characterization of hepatocellular carcinoma. Cell. 2017;169:1327-41.e23.

32. Wan S, Zhao E, Kryczek I, Vatan L, Sadovskaya A, Ludema G, et al. Tumor-associated macrophages produce interleukin 6 and signal via STAT3 to promote expansion of human hepatocellular carcinoma stem cells. Gastroenterology. 2014;147:1393-404

33. Wu J, Gao W, Tang Q, Yu Y, You W, Wu Z, et al. M2 macrophage-derived exosomes facilitate HCC metastasis by transferring a(M) $\beta(2)$ integrin to tumor cells. Hepatology. 2021;73:1365-80.

34. Zhu HF, Liu YP, Liu DL, Ma YD, Hu ZY, Wang XY, et al. Role of TGFß3-Smads-Sp1 axis in DcR3-mediated immune escape of hepatocellular carcinoma. Oncogenesis. 2019;8:43.

35. Matsui T, Nagai H, Sumino Y, Miki K. Relationship of peripheral blood CD4-positive T cells to carcinogenesis in patients with HCV-related chronic hepatitis and liver cirrhosis. Cancer Chemother Pharmacol. 2008;62:401-6.

36. Duan X, Wang M, Han X, Ren J, Huang G, Ju S, et al. Combined use of microwave ablation and cell immunotherapy induces nonspecific immunity of hepatocellular carcinoma model mice. Cell Cycle. 2020;19:3595-607.

37. Xu F, Jin T, Zhu Y, Dai C. Immune checkpoint therapy in liver cancer. J Exp Clin Cancer Res. 2018;37:110

38. Pinter M, Jain RK, Duda DG. The current landscape of immune checkpoint blockade in hepatocellular carcinoma: a review. JAMA Oncol. 2021;7:113-23.

39. Liang L, Ge K, Zhang F, Ge Y. The suppressive effect of co-inhibiting PD-1 and CTLA-4 expression on H22 hepatomas in mice. Cell Mol Biol Lett. 2018;23:58.

40. Tao LJ, Pan XY, Wang JW, Zhang L, Tao LS, Liang CZ. Circular RNA circANKS1B acts as a sponge for miR-152-3p and promotes prostate cancer progression by upregulating TGF-a expression. Prostate. 2021;81:271-8.

41. Tian Y, Li L, Lin G, Wang Y, Wang L, Zhao Q, et al. IncRNA SNHG14 promotes oncogenesis and immune evasion in diffuse large-B-cell lymphoma by sequestering miR-152-3p. Leuk Lymphoma. 2021;62:1574-84.

\section{Publisher's Note}

Springer Nature remains neutral with regard to jurisdictional claims in published maps and institutional affiliations. 